\title{
Tableau-Like Automata-Based Axiomatization for Propositional Linear Temporal Logic
}

\author{
Nikolay V. Shilov* \\ A.P. Ershov Institute of Informatics Systems \\ Novosibirsk, Russia \\ shilov@iis.nsk.su
}

\begin{abstract}
Propositional Linear Temporal Logic (PLTL) is a very popular formalism for specification and verification of computer programs and systems. This extended abstract sketches a tableau-like axiomatization for PLTL based on automata-theoretic decision procedure coupled with tableau for local model checking of the propositional $\mu$-Calculus.
\end{abstract}

Propositional Linear Temporal Logic (PLTL) is a very popular formalism for specification and verification of computer programs and systems [6, 1]. Fundamental results on decidability, model checking, and axiomatization for PLTL have become a part of the Computer Science classics [3, 9. The axiomatization issues for PLTL have been studied first on base of modal logic tradition [4. But tableau and tableau-base decision procedure for PLTL have been developed with aid of automata-theoretic technique also [10].

The talk will present sound and complete experimental tableau-like axiomatization for PLTL. This axiomatization is automata decision procedure for PTLP augmented by model checking deciding automata as a finite model. A deduction strategy within the axiomatization consists of a number of stages. These stages are sketched below along with outlines of the approach.

First we introduce the rewriting rules that eliminate negations outside literals and emulate subformulae of all until constructs $(U)$ by new propositional symbols. The rules preserve tautologies and lead to a so-called simple formulae.

Then we study a special class of automata on infinite words. An automaton in this class accepts an infinite word as soon as it enters any accepting control state. A (fairness) constraint is a set of input symbols. An infinite word meets the constraint iff all specified symbols occur finite number of times at most. The halting (termination) problem with the constraint consists in checking whether an automaton accepts every infinite word that meets the constraint; if it is the case than we say that the automaton totally accepts the constraint. We prove that the problem is decidable ${ }^{1}$.

At the next step we translate simple formulae of PTLP into automata with fairness constraint. Control states of the automata are finite sets of formulae. The main property of this translation follows: a formula is a tautology iff the automaton totally accepts the constraint. After that we consider automata as finite labeled transition systems (i.e. Kripke structures) for the propositional $\mu$-Calculus of D. Kozen $(\mu \mathrm{C})$ [5], and encode the halting problem with constrains by a particular formula of $\mu \mathrm{C}$. An automaton totally accepts a constraint iff the formula holds in some initial state of the corresponding model. In simple words: we interpret halting problem with fairness constraint(s) as the local model checking problem for some fixed formula of the propositional $\mu$-Calculus.

\footnotetext{
${ }^{*}$ The work is supported by RFBR grant N 09-01-00361-a.

${ }^{1}$ We do believe that automata-theoretic approach to decidability can exploit different variants of automata, not necessary well-known (i.e. Büchi or Rabin) automata. For example, it was the case in [8], when elementary decidability has been established for Propositional Dynamic Logic with looping and converse.
} 
Finally we adopt sound and complete tableau designed for local model checking for the $\mu \mathrm{C}$ in finite models [2] and convert it into a tableau-like axiomatization for simple formulae of PTLP.

Acknowledgement. This research was started in 2003, while author was a visiting Erskine Fellow at University of Canterbury, Christchurch, New Zealand. Arthur N. Prior (1914-1967), a founder of tense and temporal logics, was Professor of Philosophy at this university in 1952-1956. So the title of the paper could be "Designing axiomatization for Propositional Linear Temporal Logic at home of Arthur Prior". Preliminary version of this research has been published as a position paper (without proofs) in the Proceedings of International Conference TABLEAUX2005 [7. Unfortunately, this research was never presented anywhere, since the author was not granted German visa in 2005 and could not participate in TABLEAUX-2005. At present an extended version of [7] with all proofs is under review in Theoretical Computer Science.

\section{References}

[1] Clarke E.M., Grumberg O., Peled D.: Model Checking. MIT Press, 1999.

[2] Cleaveland R.: Tableau-based model checking in the propositional mu-calculus. Acta Informatica, 27:725-747, 1990.

[3] Emerson E.A.: Temporal and Modal Logic. In Handbook of Theoretical Computer Science, v.B, Elsilver and The MIT Press, 1990, p.995-1072.

[4] Gabbay D.M., Pnueli A., Shelah S., Stavi J.: On the temporal analysis of fairness. In $7^{\text {th }}$ ACM Symposium on Principles of Programming Languages, Las Vegas, p. 163-173, 1980.

[5] Kozen, D.: Results on the Propositional Mu-Calculus. Theoretical Computer Science, 27:333-354, 1983.

[6] Manna Z., Pnueli A.: The temporal logic of Reactive and Concurrent Systems. Springer-Verlag, 1991.

[7] Shilov N.V. Tableau-like Axiomatization for Propositional Linear Temporal Logic. Proc. Intern. Conf. "TABLEAUX 2005. Automated Reasoning with Analytic Tableaux and Related Methods", TABLEAUX 2005 Position Papers and Tutorial Descriptions, Ed. Bernhard Beckert, Fachberichte INFORMATIK, ISSN 1860-4471, Universitat KoblenzLandau, Institut fur Informatik, technical report n.12/2005, p.27-40. Available at http://www.uni-koblenz.de/fb4/publikationen/gelbereihe/RR-12-2005.pdf.

[8] Street R.S. Propositional Dynamic Logic of looping and converse is elementary decidable. Infor. and Control, 54:121-141, 1982.

[9] Stirling C.: Modal and Temporal Logics. In Handbook of Logic in Computer Science, v.2, Claredon Press, 1992, p.477-563.

[10] Wolper, P.: The tableau method for temporal logic: An overview. Logique et Analyse, 28:119-136, 1985 